\title{
28 Resarch Soure \\ Denoising in Early Warning of Rainfall-Induced Landslides Based on Elastic Wave Signal
}

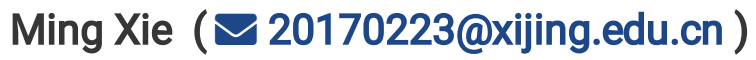

Xijing University

Jiahao Liu

Xijing University

Song Lu

Quanzhou Institute of Equipment Manufacturing Haixi Institute

Research Article

Keywords:

Posted Date: February 24th, 2022

DOI: https://doi.org/10.21203/rs.3.rs-1318476/v1

License: (c) (1) This work is licensed under a Creative Commons Attribution 4.0 International License.

Read Full License 


\section{Abstract}

The accuracy of the elastic wave signal is a key factor of elastic wave-induced landslide warning. There is too much noise in the early warning of rainfall-induced landslides, in which bending element-type piezoelectric sensors were used. At present, the mainstream method is the superposition method, which superposes multiple tested waveform data to obtain a clear waveform. However, the superposition method is limited by the number of elastic wave signals in the actual warning process, and the denoised waveform still contains high-frequency noise. A combination method combining with superposition and wavelet threshold is proposed in this paper, to improve the accuracy of the elastic waveform signal. Denoising simulation tests based on the elastic waveform signals, which collected by the bending element type piezoelectric sensor were designed to verify the combination method. The results of tests show that the combination method can effectively remove high-frequency noise and display clear waveforms, which have significant advantages in the process of rainfall-induced landslide warning using elastic waves.

\section{Introduction}

Landslides are one of the most prevalent and costly natural disasters worldwide ${ }^{1}$. Many factors influence the occurrence of landslides, with rainfall being the most common cause ${ }^{2,3}$. Traditional landslide protection methods are not always feasible due to cost and construction constraints. Thus, slope monitoring and early warning have become an efficient management strategy. Early warning of rainfallinduced landslides is frequently employed as an effective risk mitigation strategy ${ }^{4-8}$. However, the rain gauges, water content sensors and displacement sensors used in current rainfall-based landslide warnings are only sensitive to changes in the soil in their own vicinity and do not effectively monitor the

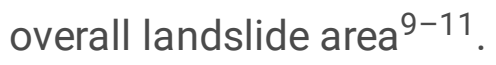

As a new type of early warning method, elastic wave-based landslide warning benefits from the fact that the elastic wave signal contains a large amount of internal physical information on the soil body, which can directly reflect the influence of boundary condition changes in the slope behavior ${ }^{12-15}$. Chen Yulong et al. verified the response characteristics of elastic wave velocity to rainfall-induced landslides by slope simulation tests ${ }^{16-19}$. The elastic wave velocity reduces as the soil water content increases, then accelerates when the slope approaches instability.

In the process of rainfall-induced slope instability, the elastic wave signals received using bending element-type sensors contain different degrees of noise ${ }^{20}$, which leads to errors in the analysis of the elastic wave signals and thus reduces the reliability of early warning. Yulong Chen et al. employed superposition denoising (SD) ${ }^{17,21}$, which involves averaging multiple measurement waveforms to minimize noise. To obtain clear waveforms for further analysis of the elastic wave signals, the data of 20 experimental waveforms were superimposed. However, the SD is limited by the amount of elastic waveform signals that can be generated, and creating elastic waveform signals too frequently can cause 
the electromagnetic coil to overheat and damage the elastic waveform signals' stability. Furthermore, the SD fails to efficiently remove high-frequency noise, and the denoised elastic wave signal is not smooth. As a result, ensuring the dependability of elastic wave-based landslide warnings requires utilizing an accurate and efficient denoising method for elastic wave signals.

The wavelet threshold denoising (WTD) proposed by Donoho and Johnstone in 1992 is of great significance in signal denoising ${ }^{22,23}$. The WTD is the most widely used denoising method because it is easy to calculate and can remove noise to a large extent while retaining the characteristics of singular information of the original signal ${ }^{24,25}$. Yangfeng Zhang et al. proposed a WTD method with Artificial Neural Network (ANN) optimized threshold for vibration sensor data in 2019, which has ideal filtering effect on vibration sensor signals ${ }^{26}$. Jiangchao Liu and Wenhua Gao combined WTD and Hilbert-Huang Transform (HHT) to denoise blast vibration signals in 2020, and the results showed that the wavelet threshold method can effectively eliminate the high-frequency noise in blast vibration signals and retain the information carried by the vibration signals themselves ${ }^{27}$. Amir Abbas Soltani and Seyyed Mohammad Shahrtash combined Decision Tree (DT) and WTD to denoise partial discharge (PD) signals of high-voltage equipment in 2020, and the results showed the superiority of the method in PD signal noise reduction for both simulated and field measurement signals ${ }^{28}$.

Because of the chance of individual elastic wave signals, this paper proposes a combination of SD and WTD for effective denoising of elastic wave signal based on reducing the number of elastic wave signals. To verify the feasibility of this method, three sets of different signals denoising simulation tests are designed and compared with SD and WTD of a single signal in this paper. At the same time, a combination of SD and WTD is used to denoise the elastic wave signals received by the bending element type piezoelectric sensor to further verify the reliability of the method. The research results will provide an important reference for the landslide warnings based on elastic waves.

\section{Digital Signal Processing}

Superposition Denoising (SD)

SD superimposes multiple sets of noise-containing data waveforms and repeatedly measures the average value of the waveforms to achieve noise reduction:

$$
y_{i}=\frac{\sum_{i=1}^{m} S_{i}}{m}
$$

where $y$ is the denoised signal; $S$ is the original signal; $m$ is the number of data sets.

The MATLAB code for the SD is provided in the literature 21. 


\section{Signal Decomposition and Reconstruction}

The decomposition and reconstruction of the wavelet is based on the tower multi-scale analysis and reconstruction of the signal proposed by Mallat in 1989. From the perspective of frequency domain, the decomposition and reconstruction of the wavelet is a band-pass filter. If the discrete sampling data of signal $f(t) \in L^{2}(R)$ are $f_{k}$ and $f_{k}=c_{0, k_{k}}$ the wavelet orthogonal decomposition formula of signal $f(t)$ is:

$$
\left\{\begin{array}{l}
c_{j, k}=\sum c_{j-1, n} h_{n-2 k} \\
d_{j, k}=\sum d_{j-1, n} g_{n-2 k}
\end{array} k=0,1,2, \cdots, N-1\right.
$$

2

where $c_{j, k}$ is the coefficient scale; $d_{j, k}$ is wavelet coefficient; $h, g$ is a pair of orthogonal mirror filter banks; $j$ is the number of levels; $N$ is the number of discrete sampling points.

The inverse operation of the decomposition process is the wavelet reconstruction process. And its formula is:

$$
c_{j-1, n}=\sum c_{j, n} h_{k-2 n}+\sum d_{j, n} g_{k-2 n}
$$

3

A signal $S$ undergoes wavelet transformation to output high-frequency components and low-frequency components, namely, Details and Approximation. By the continued wavelet transformation of the lowfrequency components, the second level of high-frequency components and low-frequency components is obtained as shown in Fig. 1.

\section{Threshold Function}

In the processing of data signals noise often appears in the form of high frequencies, while useful signals often appear in the form of low frequencies. Therefore, the high-frequency wavelet coefficients are thresholds and then the signal is reconstructed to eliminate noise. The soft and hard threshold method can reduce noise simply and efficiently by Donohn in 1992.

The hard threshold function is to discard the wavelet coefficients smaller than the threshold in different scale spaces and retain the wavelet coefficients larger than the threshold. 


$$
W_{s}\left(d_{j, k^{\prime}} \lambda_{j, k}\right)=\left\{\begin{array}{cc}
d_{j, k} & \left(\left|d_{j, k}\right| \geq \lambda_{j, k}\right) \\
0 & \left(\left|d_{j, k}\right| \leq \lambda_{j, k}\right)
\end{array}\right.
$$

where $\lambda_{j, k}$ is the threshold of each level.

The soft threshold function is to shrink the wavelet coefficients smaller than the threshold value at different scales to zero by a certain fixed amount, while retaining the wavelet coefficients larger than the threshold value.

$$
W_{s}\left(d_{j, k}, \lambda_{j, k}\right)=\left\{\begin{array}{cc}
\operatorname{sgn}\left(d_{j, k}\right)\left(\left|d_{j, k}\right|-\lambda_{j, k}\right) & \left(\left|d_{j, k}\right| \geq \lambda_{j, k}\right) \\
0 & \left(\left|d_{j, k}\right| \leq \lambda_{j, k}\right)
\end{array}\right.
$$

5

Compared with the hard threshold function, the soft threshold function processes the signal more smoothly. This study selects the soft threshold function to process the data signal.

\section{Threshold}

The choice of threshold $\lambda_{j, k}$ is also a key factor affecting the denoising effect. If $\lambda_{j, k}$ is too large, it will cause the removal of useful parts and signal distortion; in contrary, it will lead the signal to contain too much noise and fail to achieve the denoising effect.

This study selects fixed threshold:

$$
\lambda_{j, k}=\sigma_{j, k} \sqrt{2 \ln \left(N_{j, k}\right)}
$$

6

where $\sigma_{j, k}$ is the standard deviation of noise; $N_{j, k}$ is the length of each scale signal.

In the actual denoising process, the standard deviation of the noise of the signal is unknown. So, when selecting the threshold, the estimation method is used to determine the standard deviation of the noise.

$$
\sigma_{j, k}=\frac{\operatorname{median}\left(\left|c_{j, k}\right|\right)}{0.6745}
$$


where median $\left(\left|c_{j, k}\right|\right)$ is the middle value of the selected scale factor.

\section{Evaluation Indicators}

The Signal-to-Noise Ratio (SNR) and the Mean Square Error (MSE) are quoted as the evaluation indexes of the denoising effect.

\section{Signal-to-Noise Ratio (SNR)}

SNR is the ratio of the original signal to noise:

$$
S N R=10 \log _{10}\left(\frac{P_{\text {signal }}}{P_{\text {noise }}}\right)
$$

8

where $P_{\text {signal }}$ is the original signal power; $P_{\text {noise }}$ is the noise power.

$$
P_{\text {signal }}=\frac{1}{N} \sum_{i=1}^{N} f\left(x_{i}\right)^{2}
$$

9

$$
P_{\text {noise }}=\frac{1}{N} \sum_{i=1}^{N} n\left(x_{i}\right)^{2}
$$

where $N$ is the signal length; $f\left(x_{i}\right)$ is the individual element of the original signal; $n\left(x_{i}\right)$ is the individual element of the noise.

The higher the signal-to-noise ratio, the more significant the denoising effect, and the lower the signal-tonoise ratio, the less effective the denoising effect.

\section{Mean Square Error (MSE)}

MSE is used to measure the deviation of the denoised signal from the original signal:

$$
M S E=\frac{\sum_{i=1}^{N}\left|f\left(x_{i}\right)-y_{i}\right|^{2}}{N}
$$


where $y_{i}$ is the denoised signal.

The smaller the MSE, the more significant the denoising effect, and the larger the MSE, the less effective the denoising effect.

\section{Tests And Results}

In this paper, three sets of signal simulation tests are designed. Gaussian white noise with different signal-to-noise ratios is added for three different original signals (heavy sine signal, bumps signal, and doppler signal). The noisy signals are denoised using the superposition denoising with 20 signals (SD20), the superposition method with 5 signals (SD-5), the wavelet threshold denoising (WTD) and the wavelet threshold denoising after superposition with 5 signals (SD-5-WTD). The four denoising methods are compared according to the SNR and MSE, and the optimal denoising results are selected.

The steps of the simulation tests are as follows:

a) Randomly generate 20 sets of noise-added signals.

b) Denoising by the superposition method using the 20 sets of signals and calculating the SNR and MSE.

c) Five groups of signals from the 20 groups were randomly selected for denoising by the superposition method and calculating the SNR and MSE.

d) Wavelet threshold denoising was performed by randomly selecting 1 group from 20 groups of signals, and the SNR and MSE were calculated.

e) Performing wavelet threshold denoising based on the results of step b) and calculating the SNR and MSE.

f) Select the denoising method with the largest SNR and smallest MSE.

\section{Heavy Sine signal test}

Add Gaussian white noise with SNR $=20$ for the Heavy Sine signal. The decomposition level of wavelet threshold denoising is chosen as 6 layers, and the wavelet base is "db4".

Figure 2 shows the original Heavy Sine signal, the noisy Heavy Sine signal, and the Heavy Sine signal denoised by each of the four methods. The SD-5-WTD can reveal a smoother Heavy Sine signal and retain many signal details. Other methods reduce the noise to varying degrees, but the SD-5-WTD is closest to the original signal.

Table 1 shows the SNR and MSE of each method for the denoising of Heavy Sine signals. The results show that the SD-5-WTD has the highest SNR and the smallest MSE, which is the most effective method among the four denoising methods. 
Table 1

Results of SNR and MSE for Heavy Sine signal denoising.

\begin{tabular}{|lll|}
\hline Denoising Method & SNR & MSE \\
\hline SD-20 & 32.9464 & 0.0048 \\
\hline SD-5 & 26.8945 & 0.0195 \\
\hline WTD & 29.3057 & 0.0112 \\
\hline SD-5-WTD & $\mathbf{3 3 . 7 2 9 4}$ & $\mathbf{0 . 0 0 4 0}$ \\
\hline
\end{tabular}

\section{Bumps signal test}

Add Gaussian white noise with SNR $=12$ for the Bumps signal. The decomposition level of wavelet threshold denoising is chosen as 6 layers, and the wavelet base is "sym8".

Figure 3 shows the original Bumps signal, the noisy Bumps signal, and the Bumps signal denoised by each of the four methods. Due to the excessive noise added, the fluctuation of the noisy signal is obvious. However, SD-5-WTD achieved the best performance as well. The characteristics of the original signal are restored more realistically. Although SD-20 restores the overall trend of the signal, there is still too much high frequency noise.

Table 2 shows the SNR and MSE of each method for the denoising of Bumps signals. The results show that the SD-5-WTD has the highest SNR and the smallest MSE, which is the most effective method among the four denoising methods. Combined with Fig. 3, the SD-5-WTD superimposed has the most obvious denoising effect.

Table 2

Results of SNR and MSE for Bumps signal denoising.

\begin{tabular}{|lll|}
\hline Denoising Method & SNR & MSE \\
\hline SD-20 & 25.2472 & 0.0097 \\
\hline SD-5 & 18.9110 & 0.0416 \\
WTD & 19.9496 & 0.0328 \\
\hline SD-5-WTD & $\mathbf{2 6 . 0 9 8 7}$ & $\mathbf{0 . 0 0 8 0}$ \\
\hline
\end{tabular}


Add Gaussian white noise with SNR $=15$ for the Doppler signal. The decomposition level of wavelet threshold denoising is chosen as 6 layers, and the wavelet base is "sym8".

Figure 4 shows the original Doppler signal, the noisy Doppler signal, and the Doppler signal denoised by each of the four methods. The Doppler signal has much high-frequency information, which overlaps with the noise after adding noise, and the superposition denoising retains this high-frequency information to a certain extent. As the number of superimposed signals increases, the denoising effect becomes more obvious, but this is not compatible with the actual application process. The signal obtained by denoising with the SD-5-WTD is relatively smooth but missing in the high frequency part.

Table 3 shows the SNR and MSE of each method for the denoising of Doppler signals. The results show that the SD-5-WTD has the highest SNR and the smallest MSE. Combined with Fig. 4, the SD-5-WTD has significant advantages in denoising low-frequency signals, and the overall denoising effect is better than superposition denoising although it is slightly inadequate for high-frequency signals.

Table 3

Results of SNR and MSE for Doppler signal denoising.

\begin{tabular}{|lll|}
\hline Denoising Method & SNR & MSE \\
\hline SD-20 & 28.1470 & 0.00013 \\
\hline SD-5 & 21.9504 & 0.00055 \\
\hline WTD & 23.0395 & 0.00043 \\
\hline SD-5-WTD & $\mathbf{2 9 . 0 3 1 0}$ & $\mathbf{0 . 0 0 0 1 1}$ \\
\hline
\end{tabular}

\subsection{Elastic wave signal test}

To test the practical application of the SD-5-WTD for elastic wave signal, a bending element type piezoelectric transducer is used to receive 5 elastic wave signals generated from a stable source. Figure 5 shows the elastic wave signal and the denoised elastic wave signal.

The results show that the SD-5-WTD can obtain clear waveforms and enhance the accuracy of further analysis of the elastic wave signals.

\section{Conclusions}


In this paper, we add Gaussian white noise with different SNR to three signals with different characteristics in the simulation test phase and use four denoising methods to denoise the noisecontaining signals separately. The SD has a better denoising effect and clearer waveform as the number of signals increases, but it is limited by the number of signals in the actual warning process. The WTD effect of a single signal is limited, and the detail processing of the signal is not perfect. Wavelet threshold denoising after superimposing 5 signals can give a complete response to the waveform change trend and eliminate useless high frequency noise. According to the calculation results of the SNR and MSE, the SD5-WTD has the best effect.

In the elastic wave signal test stage, we collected 5 elastic wave data and processed them using wavelet threshold denoising after superimposing 5 signals. The results show that the SD-5-WTD can display clear waveforms based on overcoming the number of signals.

Our proposed combination of superposition denoising and wavelet threshold denoising optimizes the processing of elastic wave signals in elastic wave-based landslide warnings, thereby enhancing the accuracy of elastic wave signals and improving the efficiency of the elastic wave generator. Future work will consider the stability of the elastic wave generator to generate more stable elastic signals.

\section{Declarations}

\section{Data availability}

All data generated during this study are included in this article and its supplementary information files.

\section{Author contributions statement}

Methodology and Supervision, M.X., J.L and S.L.; Methodology, J.L. and M.X.; Writing-original draft, J.L.; Writing-review and editing, M.X. and S.L.. All authors have read and agreed to the published version of the manuscript.

\section{Funding}

This research received no external funding.

\section{Competing interests}

The authors declare no conflict interest.

\section{Additional information}


Correspondence and requests for materials should be addressed to M.X.

\section{References}

1. Zhou, C. \& Li, Q. Advancesin Rainfal-Induced Landslides Mechanism and Risk Mitigation. Anvances in Earth Science 24, 477-489 (2009).

2. Rahardjo, H., Nio, A. S., Leong, E. C. \& Song, N. Y. Effects of Groundwater Table Position and Soil Properties on Stability of Slope during Rainfall. J. Geotech. Geoenviron. Eng. 136, 1555-1564 (2010).

3. Li, H., Huang, R., Wu, L. \& Fang, Z. Coupling process and stability analysis of rainfall infiltration on non-homogeneous soil slopes. HYDROGEOLOGY \& ENGINEERING GEOLOGY 40, 70-76 (2013).

4. Wu, Y., Niu, R. \& Lu, Z. A fast monitor and real time early warning system for landslides in the Baige landslide damming event, Tibet, China. https://nhess.copernicus.org/preprints/nhess-2019-48/ (2019) doi:10.5194/nhess-2019-48.

5. Xu, Q., Liu, H., Ran, J., Li, W. \& Sun, X. Field monitoring of groundwater responses to heavy rainfalls and the early warning of the Kualiangzi landslide in Sichuan Basin, southwestern China. Landslides 13, 1555-1570 (2016).

6. Uchimura, T. et al. Simple monitoring method for precaution of landslides watching tilting and water contents on slopes surface. Landslides 7, 351-357 (2010).

7. Chae, B.-G. \& Kim, M.-I. Suggestion of a method for landslide early warning using the change in the volumetric water content gradient due to rainfall infiltration. Environmental Earth Sciences 66, 19731986 (2012).

8. Lollino, G., Arattano, M. \& Cuccureddu, M. The use of the automatic inclinometric system for landslide early warning: the case of Cabella Ligure (North-Western Italy). Physics and Chemistry of the Earth, Parts A/B/C 27, 1545-1550 (2002).

9. Baum, R. L. \& Godt, J. W. Early warning of rainfall-induced shallow landslides and debris flows in the USA. Landslides 7, 259-272 (2010).

10. Cannon, S. H., Gartner, J. E., Wilson, R. C., Bowers, J. C. \& Laber, J. L. Storm rainfall conditions for floods and debris flows from recently burned areas in southwestern Colorado and southern California. Geomorphology 96, 250-269 (2008).

11. Uchimura, T. et al. Precaution and early warning of surface failure of slopes using tilt sensors. Soils and Foundations 55, 1086-1099 (2015).

12. Irfan, M. \& Uchimura, T. Measuring Shear and Compression Wave Velocities in Laboratory Triaxial Tests Using Disk Shaped Composite P/S Piezoelectric Transducer. in IACGE 2013 414-421 (American Society of Civil Engineers, 2013). doi:10.1061/9780784413128.049.

13. Irfan, M. \& Uchimura, T. Modified triaxial apparatus for determination of elastic wave velocities during infiltration tests on unsaturated soils. KSCE J Civ Eng 20, 197-207 (2016). 
14. Irfan, M., Uchimura, T. \& Chen, Y. Effects of soil deformation and saturation on elastic wave velocities in relation to prediction of rain-induced landslides. Engineering Geology 230, 84-94 (2017).

15. Irfan, M. \& Uchimura, T. Development and Performance Evaluation of Disk-Type Piezoelectric Transducer for Measurement of Shear and Compression Wave Velocities in Soil. Journal of Earthquake Engineering 22, 147-171 (2018).

16. Chen, Y., Irfan, M., Uchimura, T., Wu, Y. \& Yu, F. Development of elastic wave velocity threshold for rainfall-induced landslide prediction and early warning. Landslides 16, 955-968 (2019).

17. Chen, Y. \& Uchimura, T. Early warning of rainfall induced landslides based on elastic wave velocity. Rock and Soil Mechanics 40, 3373-3386 (2019).

18. Chen, Y. \& Uchimura, T. Model tests of changes in elastic wave velocity in pre-failure soil slope due to rainfall. Chinese Journal of Rock Mechanics and Engineering 38, 2138-2150 (2019).

19. Chen, Y., Uchimura, T., Irfan, M., Huang, D. \& Xie, J. Detection of water infiltration and deformation of unsaturated soils by elastic wave velocity. Landslides 14, 1715-1730 (2017).

20. Irfan, M. Elastic wave propagation through unsaturated soils concerning early warning of raininduced landslides. (The University of Tokyo, 2014).

21. Chen, Y. Changes in elastic wave velocity in a slope due to water infiltration and deformation. (The University of Tokyo, 2016).

22. Donoho, D. L. De-noising by soft-thresholding. IEEE Trans. Inform. Theory 41, 613-627 (1995).

23. Donoho, D. L. \& Johnstone, I. M. Ideal spatial adaptation by wavelet shrinkage. Biometrika $\mathbf{8 1}, 425-$ 455 (1994).

24. Wenzhu Huang, Wentao Zhang, Tengkun Zhen, Fusheng Zhang, \& Fang Li. $\pi$-Phase-Shifted FBG for High-Resolution Static-Strain Measurement Based on Wavelet Threshold Denoising Algorithm. J. Lightwave Technol. 32, 4294-4300 (2014).

25. Wu, S. et al. Research of fetal ECG extraction using wavelet analysis and adaptive filtering. Computers in Biology and Medicine 43, 1622-1627 (2013).

26. Zhang, Y., Wei, S., Deng, N. \& Wang, W. Vibration Sensor Data Analysis Based on Wavelet Denoising. COMPUTER SICENCE 46, 537-539 (2019).

27. Liu, J. \& Gao, W. Vibration Signal Analysis of Water Seal Blasting Based on Wavelet Threshold Denoising and HHT Transformation. Advances in Civil Engineering 2020, 1-14 (2020).

28. Soltani, A. A. \& Shahrtash, S. M. Decision tree-based method for optimum decomposition level determination in wavelet transform for noise reduction of partial discharge signals. IET Science, Measurement \& Technology 14, 9-16 (2020).

\section{Figures}




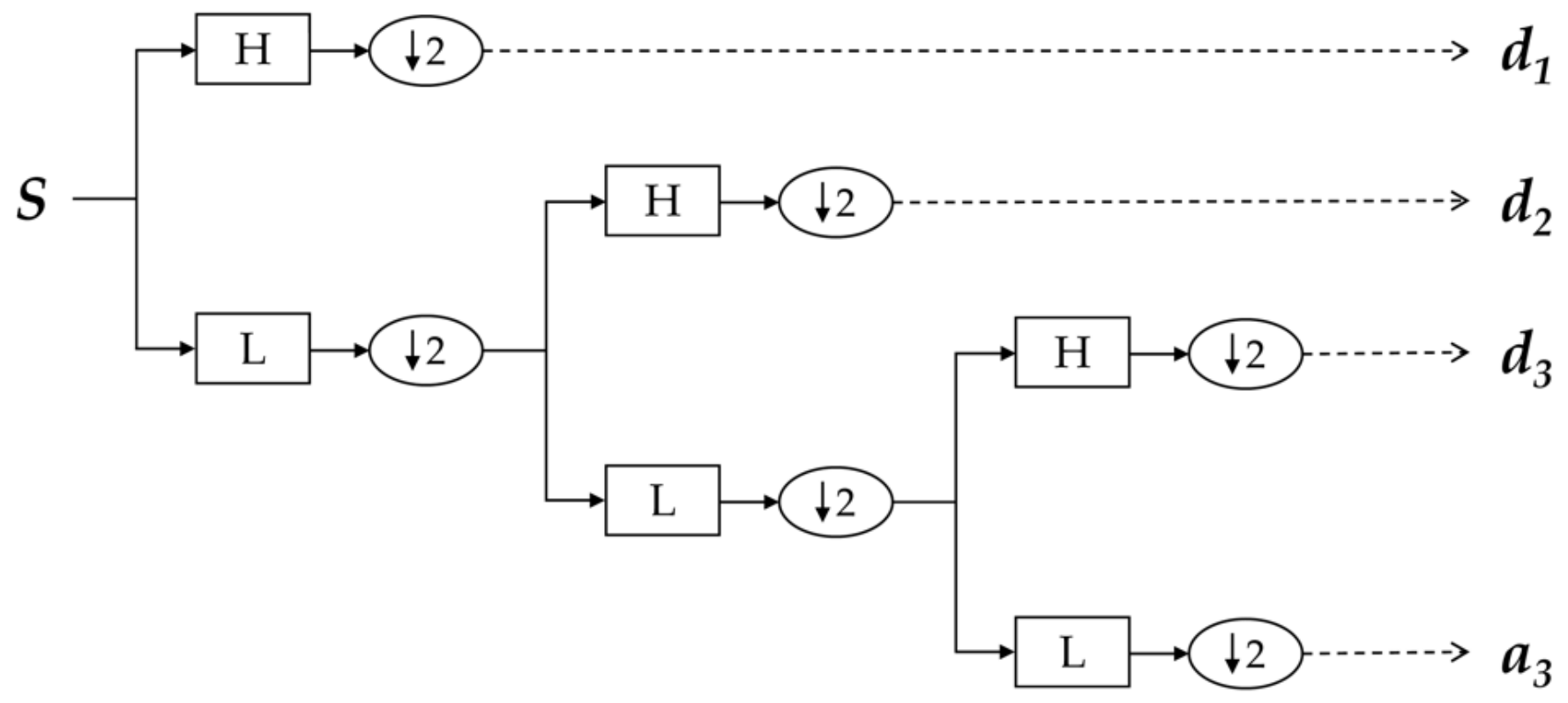

Figure 1

Diagram of wavelet decomposition. 

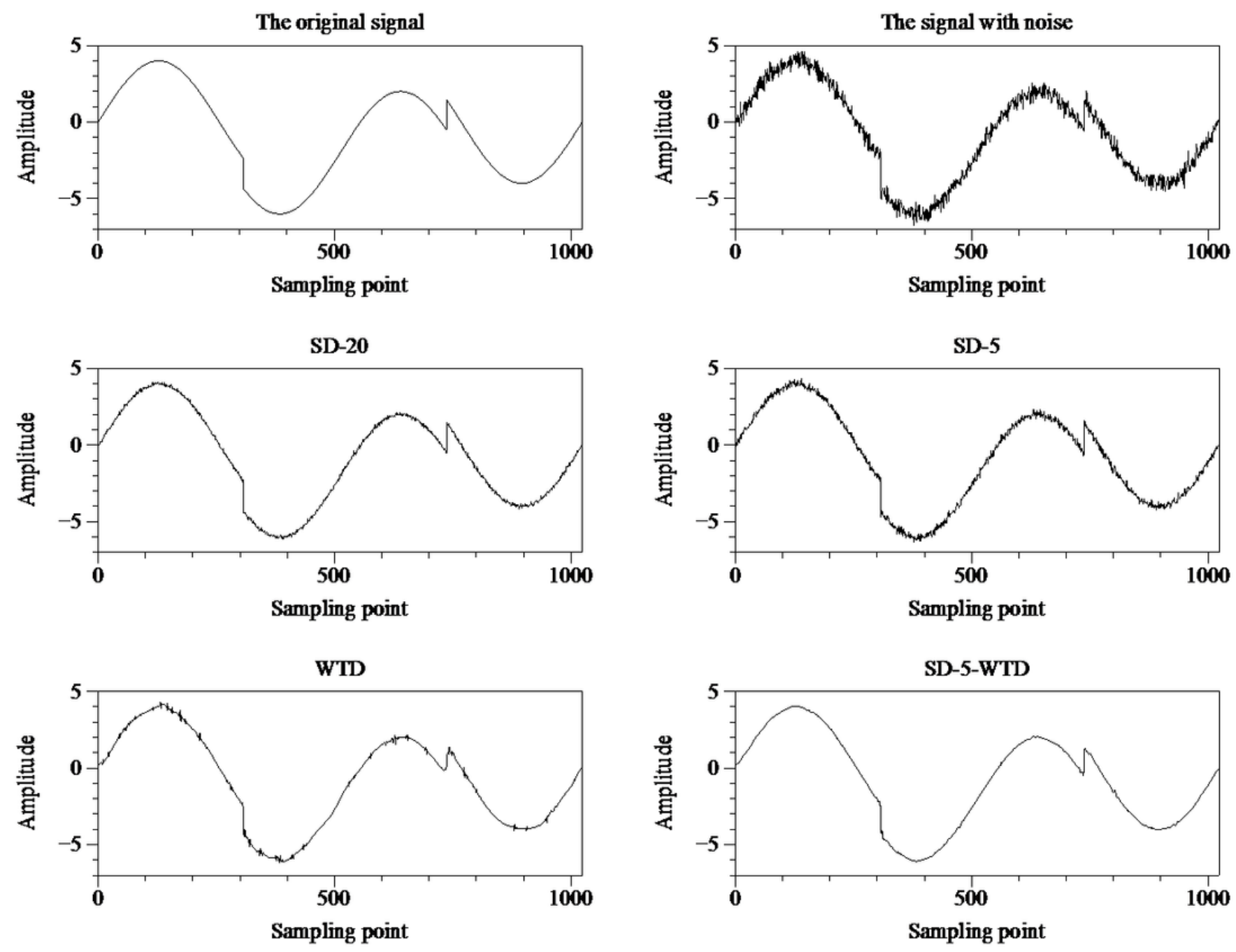

\section{Figure 2}

Results of Heavy Sine signal processing. 
The original signal

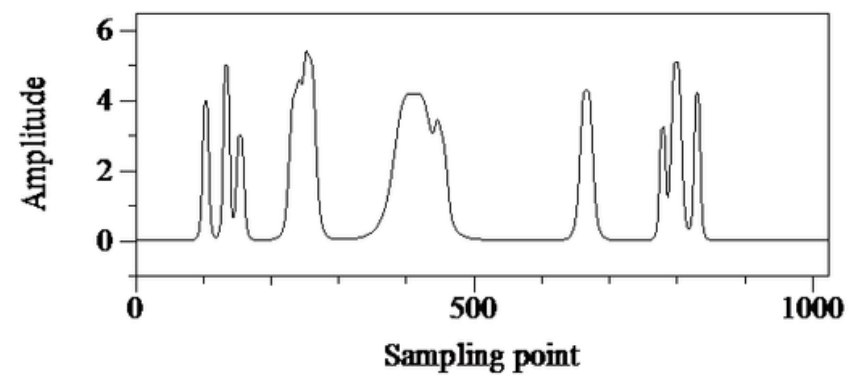

SD-20

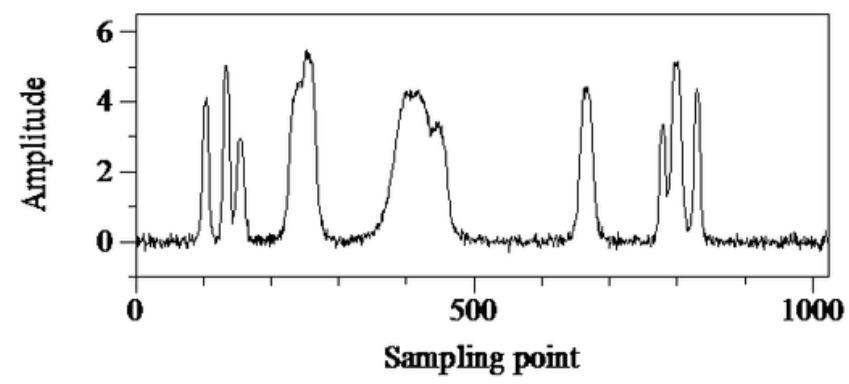

WTD

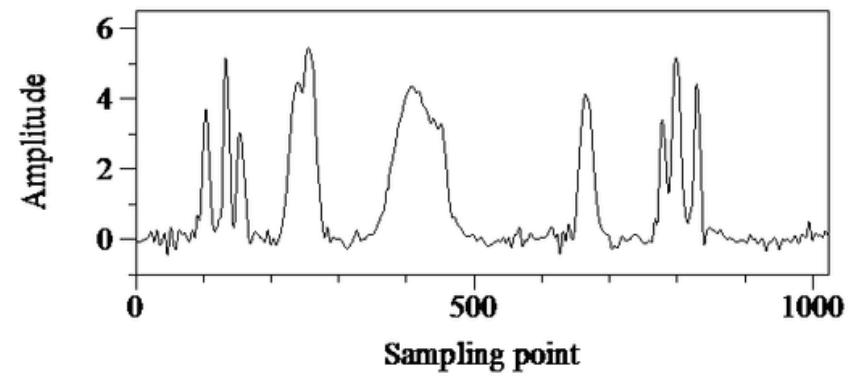

The signal with noise

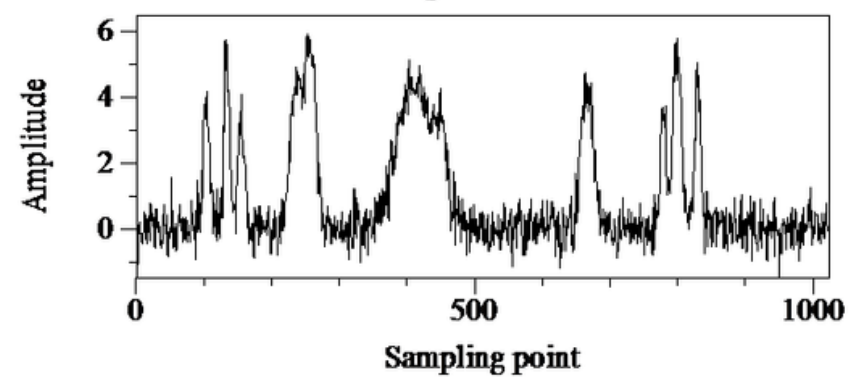

SD-5

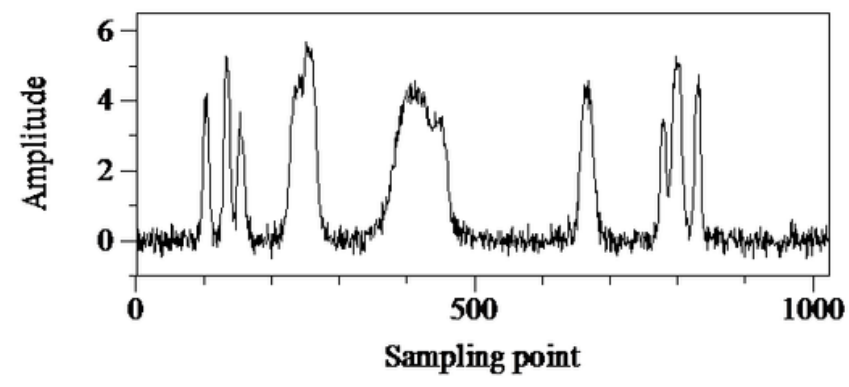

SD-5-WTD

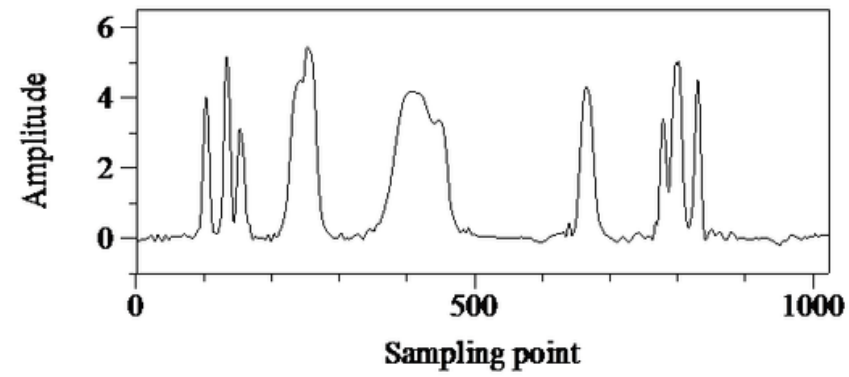

Figure 3

Results of Bumps signal processing. 
The original signal

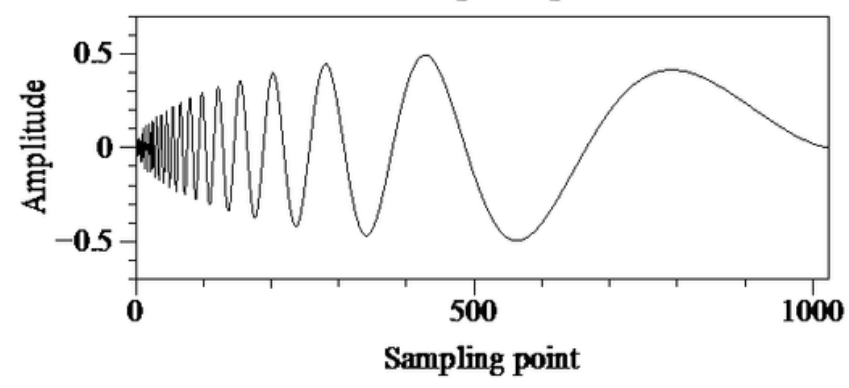

SD-20

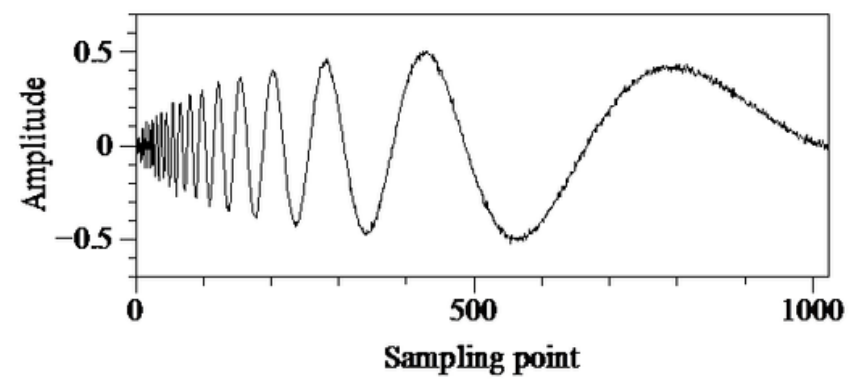

WTD

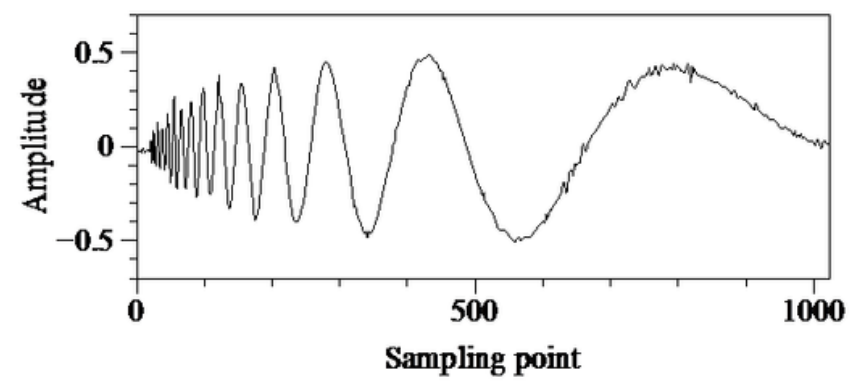

The signal with noise

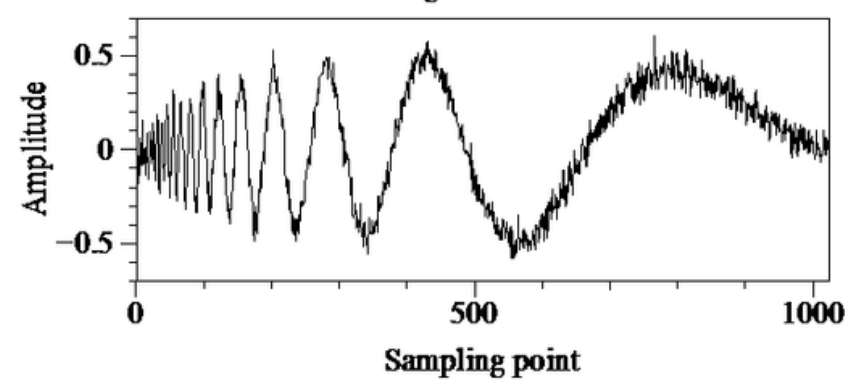

SD-5

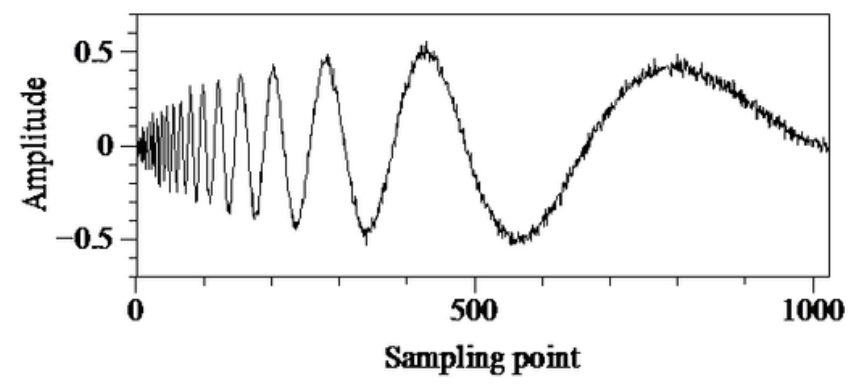

SD-5-WTD

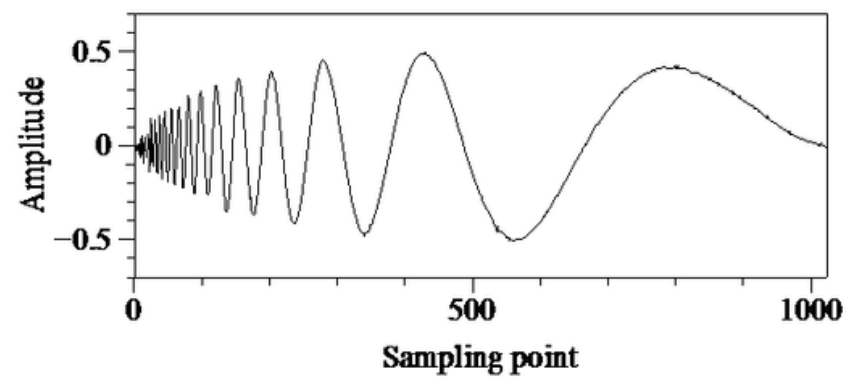

Figure 4

Results of Doppler signal processing. 

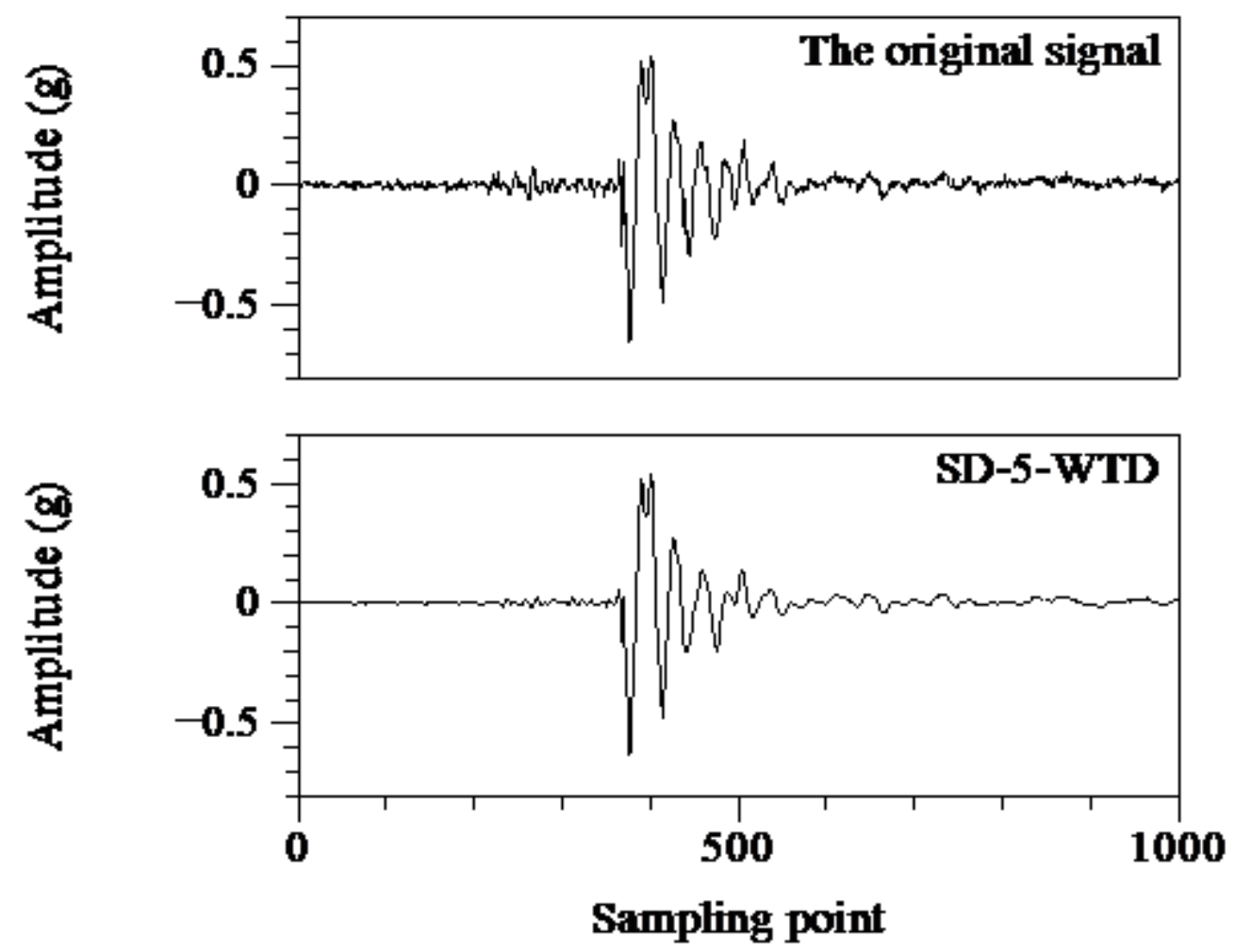

Figure 5

Results of Elastic wave signal processing

\section{Supplementary Files}

This is a list of supplementary files associated with this preprint. Click to download.

- Analysis.xlsx

- Rawdata.xlsx 